\title{
Profile of Clinical Features and Visual Loss in Diabetic Retinopathy Associated with Anaemia in Patients of Chennai City, India
}

\author{
Sowmiya Saras Manivannan ${ }^{1}$, Radha Annamalai2 ${ }^{2}$, Muthayya Muthukumar ${ }^{3}$ \\ 1, 2, 3 Department of Ophthalmology, Sri Ramachandra Institute of \\ Higher Education and Research, Porur, Chennai, Tamil Nadu, India.
}

\section{ABSTRACT}

\section{BACKGROUND}

Diabetic retinopathy is one of the commonest causes of preventable blindness. Anaemia develops earlier in diabetes patients and can hasten the development and progression of both microvascular and macrovascular complications of diabetes mellitus. The objective of the study was to identify and estimate the prevalence of anaemia in patients with diabetic retinopathy and its correlation with the progression of retinopathy, development of complications and the prognosis of vision.

\section{METHODS}

This was a retrospective study on 250 patients conducted over a period of 3 years. Comprehensive physical examination, ophthalmic evaluation using slit-lamp biomicroscopy, indirect ophthalmoscopy, fundus photography, ancillary fundus fluorescein angiography and optical coherence tomography (OCT) were performed. Blood investigations consisted of fasting blood sugar, glycosylated HbA1C, lipid profile and haemoglobin levels were estimated.

\section{RESULTS}

The prevalence of anaemia in patients with DM was $69 \%$. The prevalence of anaemia was higher in women and the age group most commonly affected was 45 to 55 years. Diabetic retinopathy was more common in men, but the severity of retinopathy was more in women. A significant association was seen between the duration of diabetes (longer than 5 years), the severity of anaemia, control of hypertension and hyperlipidaemia. The type of retinopathy most commonly associated with anaemia was moderate non-proliferative diabetic retinopathy with clinically significant macular oedema. Visual loss occurred in 16 patients.

\section{CONCLUSIONS}

Identification and treatment of anaemia can prevent the progression of retinopathy. Our study suggests that the evaluation and treatment of anaemia should be a part of initial and follow-up visits in all diabetic patients with ophthalmic features as there is a definite association between visual loss and anaemia in diabetes mellitus.

\section{KEY WORDS}

Anaemia, Diabetic Retinopathy, Maculopathy, Macular Oedema, Haemoglobin
Corresponding Author:

Dr. Radha Annamalai,

Department of Ophthalmology,

Sri Ramachandra Institute of Higher

Education and Research, Porur,

Chennai, Tamil Nadu, India.

E-mail:drradhasrmc@gmail.com

\section{DOI: $10.14260 / \mathrm{jemds} / 2021 / 785$}

How to Cite This Article:

Manivannan SS, Annamalai $R$, Muthukumar M. Profile of clinical features and visual loss in diabetic retinopathy associated with anaemia in patients of Chennai city, India. J Evolution Med Dent Sci 2021;10(45):3884-3888, DOI: 10.14260/jemds/2021/785

Submission 21-06-2021,

Peer Review 19-08-2021,

Acceptance 25-08-2021,

Published 28-12-2021.

Copyright (C) 2021 Sowmiya Saras Manivannan et al. This is an open access article distributed under Creative Commons Attribution License [Attribution 4.0 International (CC BY 4.0)] 


\section{BACKGROUND}

Diabetes mellitus is the most prevalent endocrine disorder worldwide with numerous complications. Diabetic retinopathy is a major microvascular complication of diabetes mellitus leading to visual impairment in both type 1 and type 2 diabetes mellitus. The prevalence of diabetic retinopathy significantly correlates with the duration of diabetes and glycemic control. The hallmark pathogenesis of diabetic retinopathy is microvascular occlusion and microvascular leakage. ${ }^{1}$ The systemic risk factors like anaemia, cardiovascular disease and renal failure are also involved in the pathogenesis of diabetic retinopathy. Other factors like age of onset of diabetes mellitus, gender, oral hypoglycemic agents, and insulin treatment also relate to the development and progression of diabetic retinopathy. Anaemia decreases tissue perfusion leading to hypoxic organ damage which stimulates the release of inflammatory mediators and vasoproliferative factors resulting in increased vascular permeability in the form of oedema and retinal vascular occlusive disease. ${ }^{2}$

Under normal conditions, retinal capillaries have mural cells, known as pericytes, which are responsible for the regulation of vascular caliber and control of the retinal microcirculation. These cells are found to be important in disease development since they are lost in the early stages of diabetic retinopathy. ${ }^{3}$ Endothelial damage, thickening of capillary basement membrane and loss of pericyte caused by the hyperglycemic trigger is the primary event in the development of retinopathy. Further hypoxic changes and neovascularisation lead to proliferative diabetic retinopathy. Vision loss can occur at the stage of proliferative diabetic retinopathy due to haemorrhage from fragile vessels or tractional retinal detachment as the neovascular membranes organize and contract. In certain cases, the anterior segment of the eye can also be affected with neovascularization across the angle structures leading to neovascular glaucoma. ${ }^{4}$ Diabetic macular oedema is the most common cause of visual loss secondary to diabetic retinopathy, which results in thickening of the retina and can be seen at any stage of diabetic retinopathy.

Anaemia develops early in patients with renal impairment and diabetes mellitus is the commonest cause of end-stage renal disease. Hence it is necessary to study the systemic risk factors required to delay the onset and progression of diabetic retinopathy and improving the outcome by timely intervention. Diabetic retinopathy is one of the commonest microvascular complications, others being nephropathy, neuropathy and cardiovascular disease. As of now glycosylated haemoglobin, high levels of blood sugar, elevated blood pressure are all recognized as significant risk factors. However, the association of anaemia as a risk factor in patients with type 1 and type 2 diabetes mellitus have not been studied in terms of ischemia and visual loss. The objective of the study was to identify and estimate the prevalence of anaemia in patients with diabetic retinopathy and its correlation with the progression of retinopathy, development of complications and the prognosis of vision. Our study was the first to identify the stages of retinopathy with a correlation to the levels of haemoglobin and the severity of anaemia.

\section{METHODS}

This is a retrospective, observational study performed on patients with diabetes mellitus visiting a tertiary care centre in Chennai, South India. The hospital has a well-equipped ophthalmology department and a diabetic clinic. The study was conducted over 3 years from 2018 to 2021, after obtaining institutional ethics approval (REF: CSP - MED / 21 / APR / 68 / 65). Informed consent was obtained from each patient before enrolling them in this study. The patients were reviewed every 2 - 4 weeks and more frequently based on the presentation and severity. Follow up for all the patients was done for atleast 6 months and improvement / deterioration of vision were assessed during each visit.

All diabetic patients with anaemia were evaluated in the Ophthalmology department. Both types 1 and 2 were included in our study. Grading of diabetic retinopathy was done using early treatment diabetic retinopathy study (ETDRS). According to ETDRS, patients were categorized as non- proliferative diabetic retinopathy (very mild, mild, moderate, severe and very severe) and proliferative diabetic retinopathy (mild-moderate, high risk and advanced diabetic eye disease). ${ }^{5}$ We excluded severely ill patients, patients who had known ocular morbidities associated with other systemic conditions. We noted down the duration of the condition, associated other system involvement with referrals to nephrology, cardiology and neurology, treatment for the disease and any family history of diabetes mellitus. The ophthalmic evaluation consisted of the assessment of extraocular movements, best corrected visual acuity for distance and near vision, refraction, colour vision testing, slit lamp examination, ophthalmoscopy and intraocular pressure assessment by Goldmann's applanation tonometer. Fundus examination was done with indirect ophthalmoscopy and slitlamp biomicroscopy using +78D lens and +90D lens. Optical coherence tomography was carried out for selected patients.

All data were collected and analysed to calculate the prevalence of DR in anaemic patients. The presence and type of macular oedema were noted. Visual acuity was tested during each visit using Snellen's chart and refraction was performed. During the follow-up visit, the patients were also reviewed by the physician and endocrinologist to assess improvement in the systemic illness.

Haemoglobin estimation was done by a calorimetric method. The presence of microalbuminuria was done by 24 hour urine collection. Anaemia was defined as a haemoglobin concentration of less than $13 \mathrm{gms} / \mathrm{dl}$ in men and $12 \mathrm{gms} / \mathrm{dl}$ in women. Serum lipid profile was done using fasting samples.

\section{Statistical Analysis}

All the data was collected and analysed using IBM SPSS statistics software version 23.0. In descriptive statistics, frequency analysis for data distribution and percentage analysis was done for categorical variables. Mean and standard deviation were analysed using continuous variables. Kappa coefficient was used for the correlation of test values. Statistical significance in categorical data was done using the chi-square test. A probability value of $\mathrm{P}<0.05$ was considered statistically significant. 


\section{RESULTS}

A retrospective cohort study performed on 250 patients with an established diagnosis of DM showed anaemia in $69 \%$. Among 173 patients with anaemia and DR, type 1 DM accounted for 78 patients ( $45 \%$ ) and type 2 for 95 patients (55\%). Males were affected in 50 patients (29\%) and females in 123 patients $(71 \%)$ and this was found to be statistically significant with $\mathrm{P}=0.02$. The age group affected was most frequently between 45 and 55 years (median age 47 years). Bilateral diabetic retinopathy occurred in 152 patients (88\%) with anaemia and was statistically significant with $\mathrm{P}=0.01$. Table 1 shows the epidemiological distribution of the study participants. The most common symptoms were blurred vision and floaters in both eyes. Fundus photographs of all patients were obtained, and diabetic retinopathy was based on ETDRS classification.

\begin{tabular}{|cccc|}
\hline Patient Characteristics & Variables & No of Patients & Percentage \\
\cline { 2 - 3 } Gender & Male & 50 & $29 \%$ \\
& Female & 123 & $71 \%$ \\
\multirow{2}{*}{ Types of DM } & Type 1 DM & 78 & $45 \%$ \\
& Type 2 DM & 95 & $55 \%$ \\
Laterality & Unilateral & 21 & $12 \%$ \\
& Bilateral & 152 & $88 \%$ \\
\hline \multicolumn{2}{|c|}{ Table 1. Epidemiological Distribution } \\
\hline DM-Diabetes Mellitus & \multicolumn{3}{c}{} \\
\hline
\end{tabular}

\begin{tabular}{|ccc|}
\hline Stages & No of Patients & Percentage \\
Mild NPDR & 60 & $35 \%$ \\
Moderate NPDR & 52 & $30 \%$ \\
Severe NPDR & 35 & $20 \%$ \\
PDR & 26 & $15 \%$ \\
CSME & 57 & $33 \%$ \\
Ischemic maculopathy & 15 & $9 \%$ \\
\hline \multicolumn{2}{|c|}{ Table 2. Severity of Retinopathy } \\
\hline NPDR-Non Proliferative Diabetic Retinopathy; PDR-Proliferative Diabetic \\
Retinopathy; CSME-Clinically Significant Macular Oedema \\
\hline
\end{tabular}

Sight threatening retinopathy was present in 62 out of 173 patients (36\%) and this was seen clinically as severe non-proliferative diabetic retinopathy, proliferative diabetic retinopathy or maculopathy (CSME and ischemic). Ischemic maculopathy was seen in 15 patients (9\%) and clinically significant macular oedema in 57 patients (33\%). Visual loss in diabetic retinopathy with anaemia occurred both during the active stages and with complications such as vitreous haemorrhage in 20 patients (12\%) and tractional bands and detachment in 8 patients ( $5 \%$ ). Figure 1 shows proliferative diabetic retinopathy with vitreous haemorrhage.

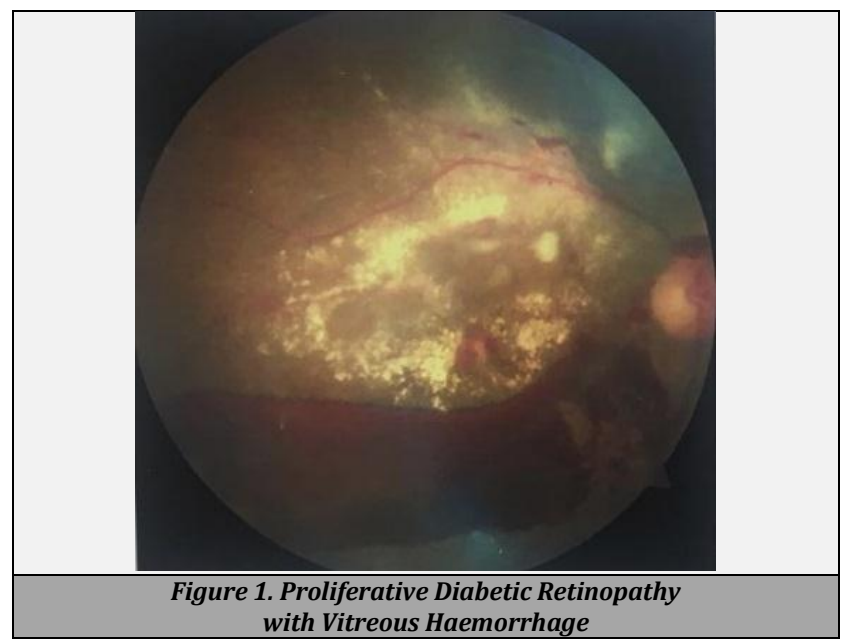

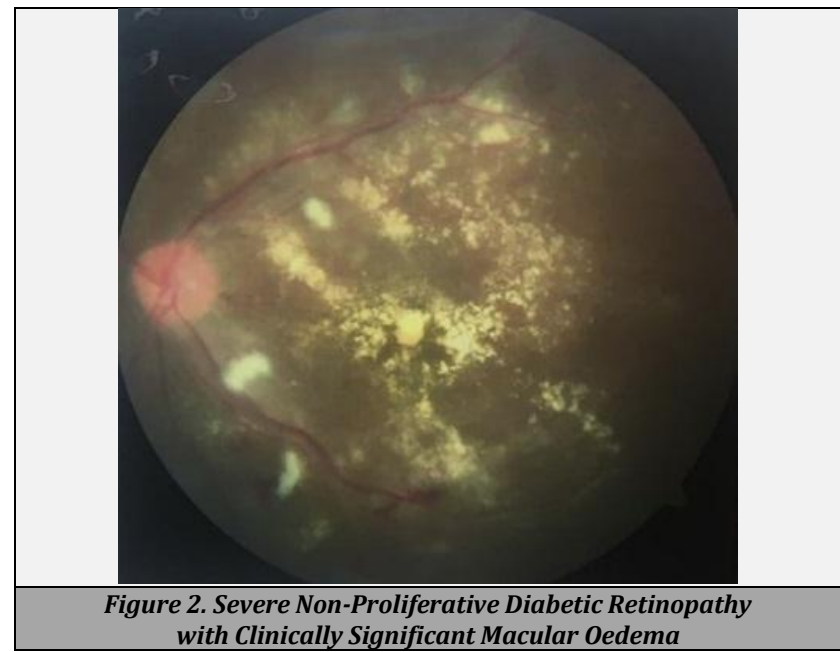

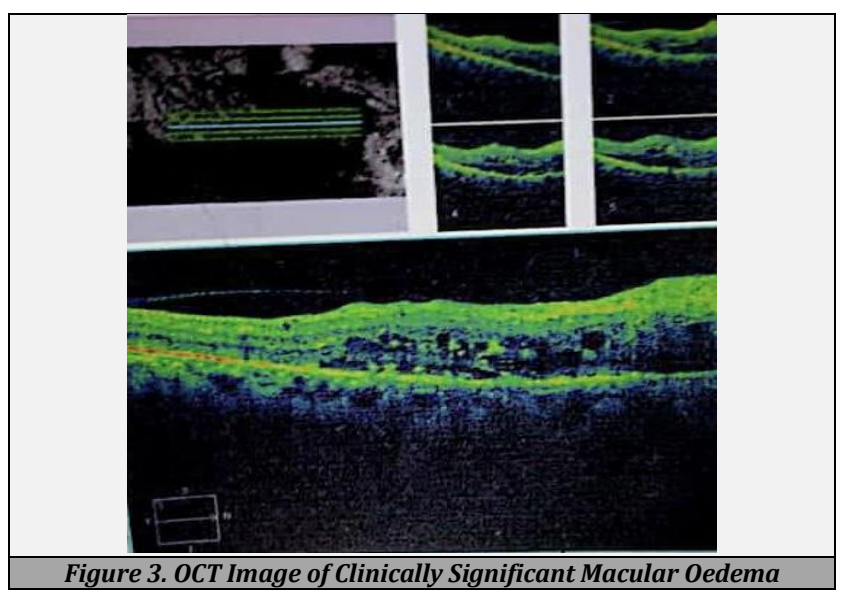

The distribution of retinopathy was seen as mild NPDR in 60 patients (35\%), moderate NPDR in 52 patients (30\%), severe NPDR in 35 patients (20\%) and PDR in 26 patients (15\%) which is depicted in table 2 . The patients with retinopathy and anaemia were younger than patients with normal haemoglobin levels with DR. Figure 2 shows severe non-proliferative diabetic retinopathy with clinically significant macular oedema.

Calculation of the association of severity of diabetic retinopathy and diabetic macular oedema with haematological parameters showed anaemia as a significant risk factor. The levels of haemoglobin were at least $12 \mathrm{gms}$ in $39 \%$ and less than 12 gms in $61 \%$ of patients. An associated raised blood pressure, triglyceride level, microalbuminuria were associated with retinopathy in varying combinations.

In terms of peripheral capillary non-perfusion, clinical changes were seen in $73 \%$ of those with anaemia. This showed a statistically significant association as capillary dropout was seen in more patients with diabetic retinopathy and anaemia. Several of these patients were on insulin treatment, had a longer duration of diabetes mellitus and associated comorbidities such as hypertension and hyperlipidaemia. In CSME, high LDL levels were noted in 42 $\%$.

Lower levels of haemoglobin had a significant association with retinal ischemia and a positive correlation with the severity of retinopathy. In terms of the progression of diabetic retinopathy and management, a rapid increase in signs is seen and required pan-retinal photocoagulation in 38 $\%$ and intravitreal anti-VEGF in $9 \%$. Another important 
feature was that we noted a marked resolution of retinal signs and an improvement in vision in our patients after strict glycaemic control in association with laser and intravitreal injections.

Our study showed a statistical significance in the correlation of retinopathy with blood sugar levels, haemoglobin levels, $\operatorname{HbA1C}(\mathrm{P}<0.05)$. Among all this anaemia was the most consistent feature as a risk factor of progression of ischemia.

The macular thickness measured by optical coherence tomography showed an increase among those with diabetic retinopathy with anaemia as compared with those who had diabetic retinopathy without anaemia. The increased central subfoveal thickness was associated with decreased vision in $93 \%(\mathrm{P}<0.04)$. Figure 3 shows an OCT picture of clinically significant macular oedema.

In our study patients, haemoglobin levels and the prevalence of anaemia at different stages of diabetic retinopathy were found to differ at different stages of diabetic retinopathy (mild, moderate, severe NPDR and PDR). Haemoglobin levels showed a mean range of 12.1, 12.3, 11, $10 \mathrm{~g} / \mathrm{dl}$ from mild NPDR to PDR.

Apart from retinopathy, ophthalmic features present were seen as snowflake cataract in $64 \%$, rubeosis iridis in 6 $\%$, neovascular glaucoma in $2 \%$, non-dilating pupil in $70 \%$ and diabetic papillopathy in $1 \%$.

At the end of 3 years of follow up, the visual loss occurred in 16 patients. Resolution of clinical signs and recovery of vision were noted in $63 \%$ of patients.

\section{DISCUSSION}

This study was performed on a cohort of patients with diabetic retinopathy. Based on our study, we found that diabetic retinopathy had a significant association with low haemoglobin levels. An overload of iron in the retinal tissue can result in cell death due to the generation of free oxygen radicals and pro-inflammatory cytokines. Diabetic retinopathy affects the microcirculation in the retina and is aggravated by hyperglycaemia. Other associated risk factors such as increased duration, poor control of HbA1C, renal disease with proteinuria, hyperlipidaemia and hypertension can worsen the retinopathy. A study by Soliman et al. had proposed that low iron levels contributed to an increase in gluconeogenic enzymes and serum glucose levels and glucose dysregulation. ${ }^{6}$ A rapid progression from background DR from a mild - to - moderate grade to a severe proliferative phase, after the development of severe iron deficiency anaemia has been reported by Chen et al. ${ }^{7}$ Balance of iron homeostasis may be of an important role in the progression of diabetic retinopathy. The maintenance of oxygen supply is of critical importance for the continuation of normal retinal function. ${ }^{8}$ Several reports have substantiated that a long duration of hypoxia which can be caused by hyperglycaemia or low haemoglobin leads to retinopathy. Iron is incorporated into heme for the transport of oxygen in the blood. Nagababu et al. reported that low iron levels enhanced red blood cell oxidative stress. ${ }^{9}$ The crucial role of oxidative stress in the development of diabetic retinopathy, wherein excessive superoxide leads to acceleration of the production of advanced glycation end-product, expression of AGE receptor, activation of protein kinase $\mathrm{C}$ isoforms, and overactivity of the hexosamine pathway have been studied extensively. ${ }^{10}$ Anaemia can accelerate the progression of retinal ischemia in patients with diabetic retinopathy. Previous studies suggested that diabetic patients have deformed erythrocytes and increased aggregation at the capillary level that could make them more fragile and susceptible to breakage, which would lower the haemoglobin levels. ${ }^{11}$ Andrews et al. showed that patients with DM and anaemia exhibited an increased expression of IL - 6 and C-reactive protein when compared to diabetic patients without anaemia. ${ }^{12}$ These inflammatory mediators cause further insult to vascular permeability and impaired normal function. Chronic inflammation can cause changes at the biochemical and molecular levels ending in retinopathy and vision loss in these patients. ${ }^{13} \mathrm{~A}$ predominant risk factor associated with the early onset of anaemia among diabetic patients is reduced GFR or elevated albuminuria levels due to destruction of interstitial fibroblasts with impaired production of erythropoietin. ${ }^{14}$ Vision threatening retinopathy is significantly increased in patients with anaemia, which is thought to be due to retinal hypoxia leading to up-regulation of growth factors such as vascular endothelial growth factor and other genes involved in neoangiogenesis, capillary permeability and apoptosis. ${ }^{15}$ Macular oedema may appear at any stage of diabetic retinopathy and is the main cause of central vision loss in diabetic patients. An evaluation of serum ferritin levels would further confirm the association between diabetic retinopathy and anaemia.

\section{CONCLUSIONS}

The study investigated the relationship between haemoglobin levels and anaemia and the occurrence of diabetic retinopathy. Our study suggests that the evaluation and treatment of anaemia should be a part of initial and follow-up visits in all diabetic patients with ophthalmic features as there is a definite association between visual loss and anaemia in diabetes mellitus.

Data sharing statement provided by the authors is available with the full text of this article at jemds.com.

Financial or other competing interests: None.

Disclosure forms provided by the authors are available with the full text of this article at jemds.com.

\section{REFERENCES}

[1] Wang W, Lo ACY. Diabetic retinopathy: pathophysiology and treatments. Int J Mol Sci 2018;19(6):1816.

[2] Baisakhiya S, Garg P, Singh S. Anemia in patients with type II diabetes mellitus with and without diabetic retinopathy. Int J Med Sci Public Health 2017;6(2):303-6.

[3] Joussen AM, Poulaki V, Le ML, et al. A central role for inflammation in the pathogenesis of diabetic retinopathy. FASEB 2004;18(12):1450-2. 
[4] Cass HG, Benitez-Aguirre PZ, Donaghue KC. Prevention and management of diabetic retinopathy in young persons with Type 1 diabetes. Diabetes Manage 2012;2(6):559-69.

[5] Wilkinson CP, Ferris FL 3rd, Klein RE, et al. Proposed international clinical diabetic retinopathy and diabetic macular edema disease severity scales. Ophthalmology 2003;110(9):1677-82.

[6] Soliman AT, De Sanctis V, Yassin M, et al. Iron deficiency anemia and glucose metabolism. Acta Biomed 2017;88(1):112-18.

[7] Chen YJ, Chen JT, Tai MC, et al. Serum iron and risk of diabetic retinopathy. Nutrients 2020;12(8):2297.

[8] Yu DY, Cringle SJ. Oxygen distribution and consumption within the retina in vascularised and avascular retinas and in animal models of retinal disease. Prog Retin Eye Res 2001;20(2):175-208.

[9] Nagababu E, Gulyani S, Earley CJ, et al. Iron - deficiency anaemia enhances red blood cell oxidative stress. Free Radic Res 2008;42(9):824-9.

[10] Galicia-Garcia U, Benito-Vicente A, Jebari S, et al. Pathophysiology of type 2 diabetes mellitus. Int J Mol Sci 2020;21(17):6275.
[11] Traveset A, Rubinat E, Ortega E, et al. Lower hemoglobin concentration is associated with retinal ischemia and the severity of diabetic retinopathy in type 2 diabetes. Journal of Diabetes Research 2016;2016:3674946.

[12] Andrews M, Arredondo M. Ferritin levels and hepcidin mRNA expression in peripheral mononuclear cells from anemic type 2 diabetic patients. Biological Trace Element Research 2012;149(1):1-4.

[13] Roy S, Kern TS, Song B, et al. Mechanistic insights into pathological changes in the diabetic retina: implications for targeting diabetic retinopathy. Am J Pathol 2017;187(1):9-19.

[14] Awofisoye Ol, Adeleye JO, Olaniyi JA, et al. Prevalence and correlates of anemia in type 2 diabetes mellitus: a study of a Nigerian outpatient diabetic population. Sahel Med J 2019;22(2):55-63.

[15] Grimm C, Wenzel A, Groszer M, et al. HIF - 1 - induced erythropoietin in the hypoxic retina protects against light - induced retinal degeneration. Nature Medicine 2002;8(7):718-24. 